\title{
Real-time visualisation of residential load flexibility for advanced demand side management
}

DOI:

10.1109/MELCON.2018.8379090

\section{Document Version}

Accepted author manuscript

Link to publication record in Manchester Research Explorer

\section{Citation for published version (APA):}

Ponocko, J., Cai, J., Sun, Y., \& Milanovic, J. V. (2018). Real-time visualisation of residential load flexibility for advanced demand side management. In 2018 19th IEEE Mediterranean Electrotechnical Conference (MELECON) https://doi.org/10.1109/MELCON.2018.8379090

\section{Published in:}

2018 19th IEEE Mediterranean Electrotechnical Conference (MELECON)

\section{Citing this paper}

Please note that where the full-text provided on Manchester Research Explorer is the Author Accepted Manuscript or Proof version this may differ from the final Published version. If citing, it is advised that you check and use the publisher's definitive version.

\section{General rights}

Copyright and moral rights for the publications made accessible in the Research Explorer are retained by the authors and/or other copyright owners and it is a condition of accessing publications that users recognise and abide by the legal requirements associated with these rights.

\section{Takedown policy}

If you believe that this document breaches copyright please refer to the University of Manchester's Takedown Procedures [http://man.ac.uk/04Y6Bo] or contact uml.scholarlycommunications@manchester.ac.uk providing relevant details, so we can investigate your claim.

\section{OPEN ACCESS}




\title{
Real-time Visualisation of Residential Load Flexibility for Advanced Demand Side Management
}

\author{
Jelena Ponoćko, Student Member IEEE, Jiawei Cai, Yusong Sun, and Jovica V. Milanović, Fellow IEEE \\ School of Electrical and Electronic Engineering \\ The University of Manchester \\ Manchester, UK
}

\begin{abstract}
Demand side management has been recognized as one of the potentially significant flexibility resources whose deployment will be necessary for the operation of future power transmission, and probably more so, distribution networks. With this in mind the greater observability of the demand is becoming an important requirement, not only for keeping the network performance parameters within their limits, but also for assessing the availability and flexibility of demand at any given time. Besides forecasting total power demand at lower aggregation levels to facilitate the balancing of demand and available distributed generation, the key information for flexible network operation is the amount of (time-varying) controllable loads in the geographical area of interest. This paper introduces a two-module graphical user interface (GUI) for the operator of the future distribution network that provides in a user friendly form the information on real-time demand forecasting and demand composition. The GUI presents, both graphically and numerically, the forecasted and actual active and reactive power demand and the composition of the forecasted demand in terms of different categories (resistive loads, motors, lighting, etc.) of the load and total controllable and uncontrollable load. The information is provided, based on the user requirements, either a half hour or 24 hours ahead.
\end{abstract}

Index Terms- Demand-side management, distribution network, smart meter, neural networks, graphical user interface

\section{INTRODUCTION}

Demand side can be ultimately observed as one of the distributed energy resources (DER), as it may provide the distribution network with the spatially distributed flexibility necessary for meeting the different requirements (peak shaving, congestion mitigation, etc.) of the distribution network operator (DNO). An important advantage of aggregated load, whether it is residential, industrial or commercial, is that it can respond quickly, and contains a large number of smaller devices, whose individual variations in power demand have a low effect on the network [1]. Considering the large number of small individual loads, an aggregator will have an important role in interfacing between a substantial number of end-users and the electricity market. In the UK, for example, most users participating in demand response (DR) are contracted via an aggregator. National Grid, the transmission system operator in the UK, has set a goal of contracting $30-50 \%$ of balancing capacity from demand side by 2020 , with an estimated DR potential of around $9.8 \mathrm{GW}$ [2]. Although the main participants in DR have been large industrial users [3], there is an insufficiently explored flexibility potential in residential areas. In some countries, such as the US, it has been estimated that residential DR could bring up to half of the total peak reduction [4].

One of the necessary conditions for the successful deployment of DR programs in the residential sector is better observability of the end-users, which is made possible by the use of smart meters (SMs). On the one hand, smart metering will facilitate awareness of consumers about their daily consumption and enable them to make savings by reacting to price signals or various types of incentives triggered by their electricity supplier. On the other hand, SM data will provide information to the DNO about individual load profiles, enabling more advanced profiling of consumers in different areas and at different levels of aggregation. Furthermore, with the development of per-appliance (sub) metering (which has been reported in several pilot sites $[4,5]$ ), it would be possible to observe the daily usage of different appliances, which could facilitate the design and execution of the DR programs. By aggregating the sub-metering data of a large number of users, one could assess the actual flexibility (controllability) of the aggregated load at a substation, for example. If the flexible loads were aggregated at each substation (or any other aggregation point) in a network, it would be possible to have an overview of the spatial distribution of demand side flexibility as one of the DER in the network.

This paper introduces a Graphical User Interface (GUI) for advanced demand profiling (ADP) of the residential load, as a decision making tool for a DR responsible party (e.g. an aggregator or DNO). ADP presents a combination of short or mid-term (half hour or 24 hour ahead) total active/reactive demand forecasting and decomposition of the forecasted demand at the aggregation point. Load composition is given as time-changing participation (in per unit - p.u.) of each of the six pre-defined categories within the total active and reactive load, as illustrated in Fig. 1 and 2, respectively. It can be obtained by aggregating the sub-metering data from the endusers' premises, provided that all users have the sub-metering feature enabled [6]. In cases where only limited or no sub- 
metering is available, the aggregated demand can be decomposed following the methodology introduced in $[7,8]$ and decomposed daily loading curves can be produced. This methodology $([7,8])$ estimates the composition of the forecasted demand based on limited data and the use of artificial neural networks (ANN). It was shown in [8] that even with a very limited number of end-users who have submetering, it is still possible to decompose the demand of all the users in the aggregation with a high level of confidence.

\section{DEMAND PROFILING OF RESIDENTIAL LOAD}

Total demand in this paper is decomposed into 6 load categories, which are defined as: single-phase constant torque induction motors (CTIM), single-phase quadratic torque induction motors (QTIM1), controllable resistive loads $\left(\mathrm{R}_{\mathrm{C}}\right)$, uncontrollable resistive loads $\left(\mathrm{R}_{\mathrm{UC}}\right)$, switch-mode power supply (SMPS) loads and Lighting. Each residential appliance is assigned to one of these categories, as shown in Table I. Although the main information for the DR responsible party is how much controllable (curtailable/shiftable) demand there is during the day, information about the individual load categories' shares can be very useful for analyzing the effect of DR on the overall network performance, as discussed in [9].

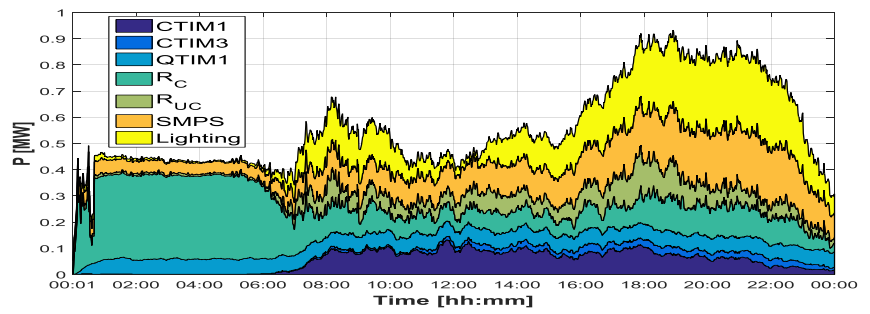

Figure 1. Active demand composition (in MW) of aggregated 1000 residential end-users over 24 hours

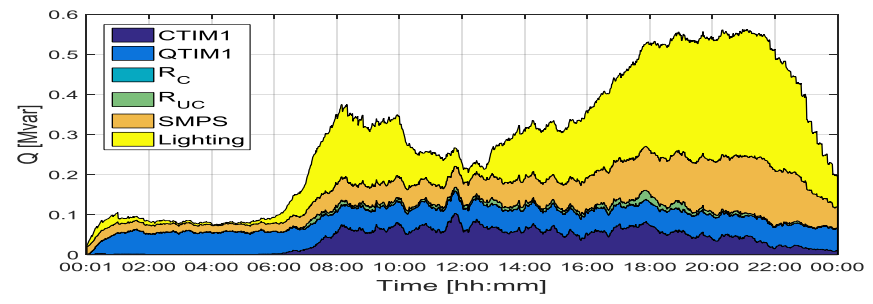

Figure 2. Reactive demand composition (in Mvar) of aggregated 1000 residential end-users over 24 hours

The full list of appliances was adopted from the residential load model given in [5]. Furthermore, categories are grouped into controllable and uncontrollable, based on their ability to be curtailed/shifted in time. Therefore, the amount of controllable (flexible) load can be obtained by adding the demand of controllable load categories. Following the methodology discussed in [9], load categories are defined based on groups of loads with similar voltage-dependent steady-state and dynamic load characteristics. Demand decomposition can therefore provide information on the amount and type of the controllable load at any given time (from close to real time to a specified point in time in the future). For any type of direct load control or incentive-based DR program, an estimation/prediction of demand composition at the aggregation point could show if the DR potential (flexibility) of the aggregated consumption is sufficient for the needs of the program (peak shaving, for example), or if some additional measures are necessary (for example, increasing the incentives for the end-users).

TABLE I. LOAD CATEGORIES AND CORRESPONDING TYPES OF APPLIANCES

\begin{tabular}{c|c|c}
\hline $\begin{array}{c}\text { Load } \\
\text { controllability }\end{array}$ & $\begin{array}{c}\text { Load } \\
\text { categories }\end{array}$ & Residential appliances \\
\hline \hline \multirow{4}{*}{ Controllable } & CTIM1 & $\begin{array}{c}\text { Dish washer, tumble dryer, washing } \\
\text { machine, washer-dryer, vacuum cleaner }\end{array}$ \\
\cline { 2 - 3 } & QTIM1 & $\begin{array}{c}\text { Chest freezer, fridge-freezer, fridge, } \\
\text { upright freezer }\end{array}$ \\
\cline { 2 - 3 } & $\mathrm{R}_{\mathrm{C}}$ & $\begin{array}{c}\text { Water heater, electrical shower, storage } \\
\text { heater, electrical space heating }\end{array}$ \\
\hline \multirow{4}{*}{ Uncontrollable } & $\mathrm{R}_{\mathrm{UC}}$ & Iron, hob, oven \\
\cline { 2 - 3 } & SMPS & $\begin{array}{c}\text { Answer machine, CD player, Clock, } \\
\text { telephone, high fidelity (HiFi) } \\
\text { appliances, Fax machine, PC, printer, } \\
\text { TV, VCR-DVD, receiver, microwave }\end{array}$ \\
\cline { 2 - 3 } & Lighting & Lighting \\
\hline
\end{tabular}

The overall ADP methodology has been initiated within the UK EPSRC project "Autonomic power systems" and EU FP7 SUSTAINABLE project and fully developed within the EU H2020 NOBEL GRID project as a micro-service of the integrated NOBEL GRID Consumer Profiling Framework. It incorporates two functionalities: mid-term (day ahead) and short term (half hour ahead) demand forecasting (DF) and demand decomposition (DD), the latter relying on the former. . The reason for this interdependence is that the necessary input for demand decomposition is total active and reactive demand, as described in [7]. As most of the DR programs are planned based on the forecasted flexibility of the end-users (e.g. in [10]), the information about demand composition should also be given in advance, most commonly one day ahead. Therefore, as a necessary step before DD, information about forecasted active and reactive demand has to be obtained first. The forecast is done at the aggregation point, which is usually the substation supplying the consumers participating in the DR programme. Other scenarios, where the aggregation involves consumers scattered around a geographical (and electrical network) area, are also possible.

Demand forecasting is done at the aggregation level, either at the substation or for a group of scattered customers belonging to the same aggregator, and don't necessarily have to be connected to the same LV substation. The forecast is performed either a day or half hour ahead and it has two main applications:

- distribution network operation, where the forecasted load is used as the necessary information for balancing between demand and available generation, and 


\section{ACCEPTED VERSION OF THE PAPER}

- input for the DD module which uses the total demand prediction to forecast demand composition.

The former application is complemented by a renewable energy sources (RES) forecast, which allows low carbon operation of the distribution network; the latter application actively supports the DR programs, by providing information on forecasted amount and type of controllable loads of the endusers.

\section{METHODOLOGY}

The information flow between the two modules of the ADP and the GUI is illustrated in Fig. 3. The input data is first sent to the DF module, whose results (forecasted active and reactive demand) are then fed to the DD module, for finally obtaining the composition of the forecasted demand, as shown in Fig. 1 and 2.

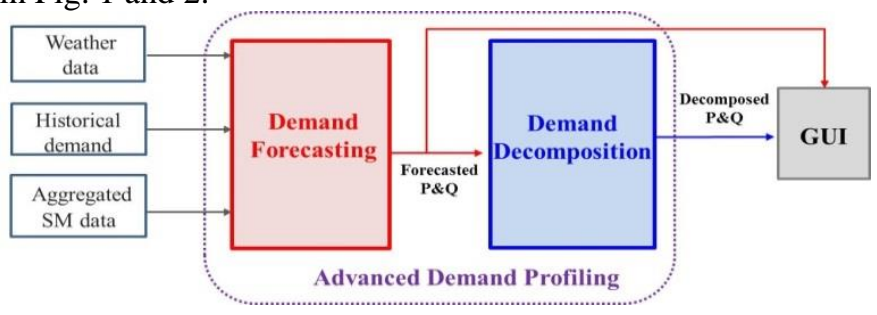

Figure 3. Flowchart of the information flow

\section{A. Demand Forecasting}

The DF module is based on the use of ANN, following methodology for base load forecasting introduced in [9], which uses historical demand data (active/reactive load) and weather data (temperature, humidity and wind speed) for training the ANN, and most recent historical demand and forecasted weather data for obtaining the half hour or day ahead demand forecast. The training of the ANN is presented in Fig. 4, where three rows for weather data correspond to temperature, humidity and wind speed, and day type refers to a working/non-working day (1/0). Once the ANN is trained, the input data should be in the same format as the training inputs. In order to retain acceptable accuracy of the DF, historical data is regularly updated, with the fixed time window of the training data. This means that the ANN for DF (for simplicity, referred here as DFANN) is always trained with the same size of historical data (in this example, 3 months) by dismissing the oldest samples every time the training data is updated with new measurements, for example daily or weekly.

Besides historical demand and the weather data necessary for training the DFANN, the update rate of weather forecast (input) data is also important. The DF module was tested using test data from an actual test site (substation), where historical data measurements of active demand (with half hour resolution) and corresponding weather (one hour resolution) were available. The weather forecast for the next 24 hours would be updated once/twice a day, which affected the accuracy of results. Fig. 5 illustrates the importance of input data update on the accuracy of day ahead DF. Datasets 1 and 2 represent forecasting outputs where weather data is updated every 12 hours and 24 hours, respectively. The relative errors (in \%) based on the forecasted and actual (measured) load values are given over a 36 hour period (72 time steps).

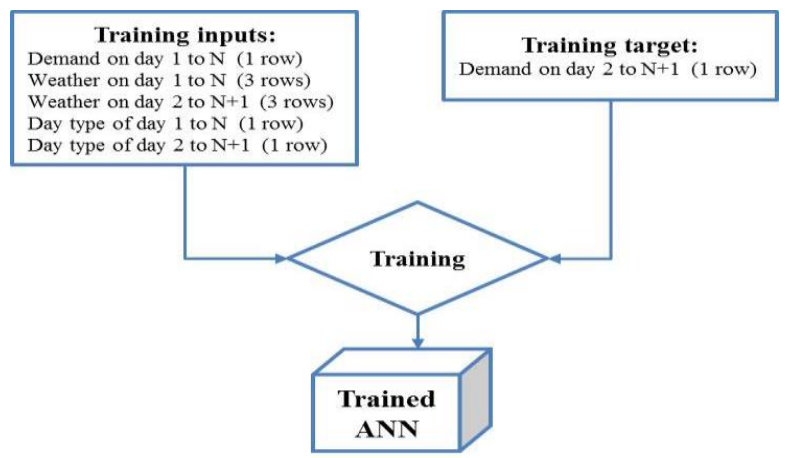

Figure 4. Training process of the DFANN

Between time steps 14 (corresponding to 7 a.m.) and 38 (corresponding to 7 p.m.), dataset 1 is forecasted based on the updated weather data, while dataset 2 still uses the old weather forecast (which only gets updated at 7 p.m.), resulting in different accuracy, as highlighted in the frame in Fig. 5.

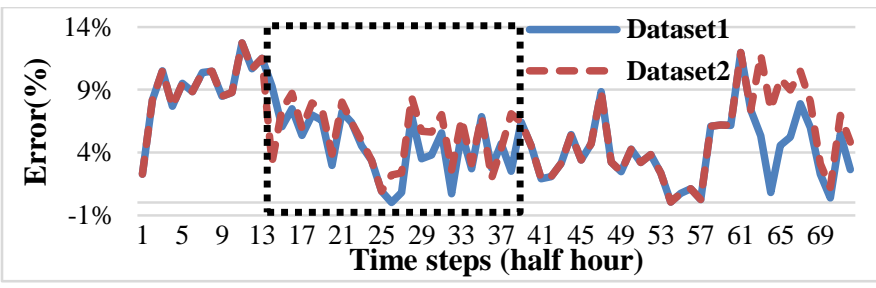

Figure 5. Relative error of demand forecasting for datasets 1 and 2 over 36 hours (72 time steps)

After this period, during the next 24 steps (12 hours), both datasets are forecasted based on the same weather data, which is why the errors are the same during that period. In order to show the dependence on the weather update, Table II presents the most probable relative errors (MPRE) for day ahead and half hour ahead forecasting, for datasets 1 and 2 .

TABLE II. THE MOST PROBABLE RELATIVE ERROR FOR DIFFERENT DEMAND FORECASTING CASES

\begin{tabular}{c|c|c|c|c}
\hline \multirow{2}{*}{ Case } & \multicolumn{2}{|c|}{ Dataset 1 } & \multicolumn{2}{c}{ Dataset 2 } \\
\cline { 2 - 5 } & Day & Half hour ahead & $\begin{array}{c}\text { Day } \\
\text { ahead }\end{array}$ & Half hour ahead \\
& ahead & & $\mathbf{6 . 7 4 \%}$ & $2.40 \%$ \\
\hline MPRE & $\mathbf{4 . 7 1 \%}$ & $2.38 \%$ & . & \\
\hline
\end{tabular}

As seen in the table, the weather update shows a higher impact on the accuracy of day ahead forecasting than of half 


\section{ACCEPTED VERSION OF THE PAPER}

hour ahead forecasting.(Note: two different DFANNs are generated for day ahead and half hour ahead forecasts.) The reactive demand forecast can be analyzed in a similar way, as long as the reactive power measurements (or power factor values, as in this test site's data) are available.

\section{B. Demand Decomposition}

The diagram in Fig. 6 presents the ANN based methodology for load decomposition in the case of a smart metering system where only some consumers in the aggregation area have sub-metering enabled. This methodology is described in more detail in [7]. A separate ANN is used for load decomposition (here, referred to as DDANN), with settings identical to those suggested in [8]. In this case, available sub-metering data is used for training the ANN, using total (aggregated from SMs) active and reactive power as input data and participation of the six categories (in p.u.) as the target data (blocks 1-3 in the diagram).

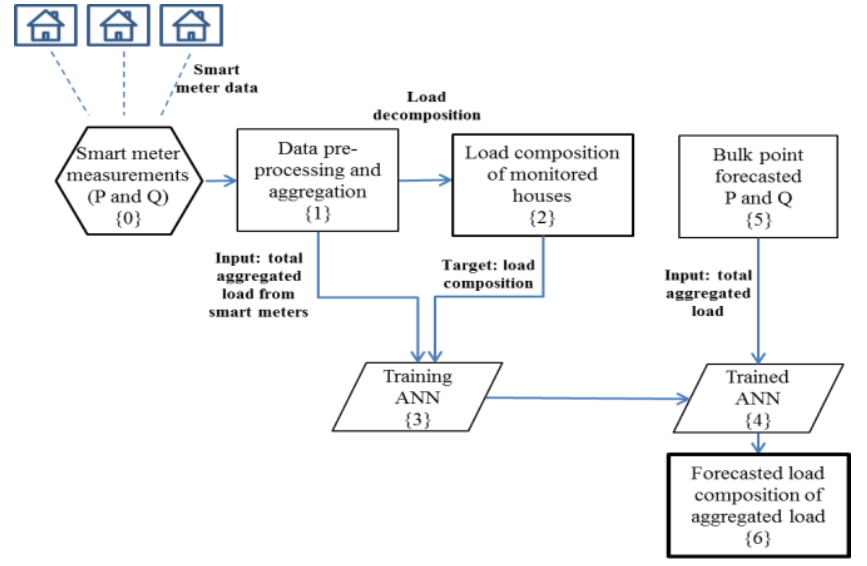

Figure 6. Flowchart of demand decomposition

Assuming a minute-based resolution of measurements, the training process is performed over 7 days data, which includes inputs - real power $(P)$ and reactive power $(Q)$ measurements, giving $7 \times 1440=10080$ samples for each of the variables, presented in a matrix form as follows:

$$
\text { Input }=\left[\begin{array}{ccccc}
P_{1} & \cdots & P_{i} & \cdots & P_{10080} \\
Q_{1} & \cdots & Q_{i} & \cdots & Q_{10080}
\end{array}\right]
$$

Target data for the ANN training is defined as $W$, and presents the participation of each of the six load categories (in p.u.) within the total active demand (the first row of the Input in (1). If in time step $i$, the active load of category $j$ equals $P_{j i}$, the participation of that category is given as:

$$
w_{j i}^{P}=\frac{P_{j i}}{P_{i}}
$$

where $P_{i}$ is the total active load in time step $i(i=1 \div 10080)$ Target data can then be presented in matrix form as given in (3). The participation of each category in reactive load is given by expression (4), where $\varphi_{j i}$ and $\varphi_{i}$ are phase angles of category $j$ and total load in time step $i$, respectively. Once trained, the DDANN (block $\{4\}$ ) uses forecasted total active and reactive load at the substation as the input, giving its load composition as the output (block $\{6\}$ ), in the same format as the Target defined in (3). The forecasted $\mathrm{P}$ and $\mathrm{Q}$ are the results of the DF module. In cases where there is no submetering data available, the DDANN can be trained with probabilistically derived data (by Monte Carlo simulations), as described in [9].

$$
\begin{gathered}
\text { Target }=\left[\begin{array}{ccc}
w_{1,1}^{P} & \cdots & w_{1,10080}^{P} \\
w_{2,1}^{P} & \cdots & w_{2,10080}^{P} \\
\vdots & \cdots & \vdots \\
w_{6,1}^{P} & \cdots & w_{6,10080}^{P}
\end{array}\right] \\
w_{j i}^{Q}=\frac{Q_{j i}}{Q_{i}}=\frac{P_{j i} \tan \left(\varphi_{j i}\right)}{P_{i} \tan \left(\varphi_{i}\right)}=w_{j i}^{P} \frac{\tan \left(\varphi_{j i}\right)}{\tan \left(\varphi_{i}\right)}
\end{gathered}
$$

\section{GRAPHICAL USER INTERFACE}

In order to present the ADP output in a user-friendly way, two Graphical User Interfaces (GUIs) have been developed, for DF and DD. Print-screens of the two GUIs are presented in Fig. 7 and 8, showing the ADP results using the historical total active/reactive demand and weather data from the aforementioned test site. As the site had no sub-metering data, the training data for DDANN was generated using Monte Carlo simulations and statistical data about the load composition in residential areas, similar to the approach described in [9]. Both GUIs (for the DF and DD module) offer day ahead and half hour-ahead forecasting. As both GUIs are updated with new measurements arriving in real-time, the actual total demand (from substation measurements) and actual demand composition (resulting from the DDANN taking measured $\mathrm{P}$ and $\mathrm{Q}$ data as input), are presented, enabling real-time comparison and error calculation between the forecasted and actual values. The two GUIs "communicate" with each other, the output of the DF being used as the input for DD.

Although DF is an on-line application, there is a certain time delay between the input data acquisition and the output forecasted active/reactive load. Similarly, additional delay will be introduced with DD, as it relies on the output of DF and takes some time for the simulations. The delays will depend on the data latency of the communication system responsible for gathering the measurements, data pre-processing time and also from the computer speed. With the 64-bit operating system and $3.40 \mathrm{GHz}$ processor used in this example, delay introduced by the DF module was 5 seconds, while the delay caused by the DD module was 7 seconds, giving 12 seconds of total delay for the overall ADP process. 


\section{ACCEPTED VERSION OF THE PAPER}

Fig. 7 represents the GUI for DF, where different numbered parts of the interface have the following function/meaning:

- 0: Activate/deactivate button serves to start or interrupt the GUI. (Note: The DFANN is first trained with the most recent, in this case 3 months-long, historical weather and demand data. Once trained, only the last half hour/24 hour demand and half hour/24 hour ahead weather forecast data is needed as the input to perform the half hour/24 hour ahead demand forecast.);

- 1 and 2: Bar plots showing the time change of the key performance indicators (KPIs), i.e. relative errors between the forecasted and actual values for real and reactive demand, respectively. Red bars refer to the half-hour ahead forecasting errors, and blue ones to the day-ahead forecasting errors, which are usually higher. The presented KPIs are illustrative - additional indicators, for example mean absolute percentage error (MAPE) [9], can be added;
- 3: The 'Latest status' shows the current time (here, 24/09/2015 at 04:00), and current (real-time) measured active and reactive demand at the substation;

- 4 and 5: Graphs presenting day ahead (in blue) and half hour-ahead (in red) forecasted active and reactive demand, respectively, together with the actual (measured) demand (in black). The vertical green line refers to the current time - as the time changes, the demand curves move to the left, while the green line stays fixed. The curves will move with the same time resolution as the resolution of the measurement data, with some time delay, as mentioned. Therefore, the curves on the right side of the green line refer to the forecasted demand, while those on the left side correspond to the most recent historical data, measured and forecasted;

- 6 and 7: Numerical values of day-ahead and half hourahead forecasted active and reactive demand, respectively, which were forecasted for the current time, followed by the calculated relative error based on the actual (measured) demand at the current time;

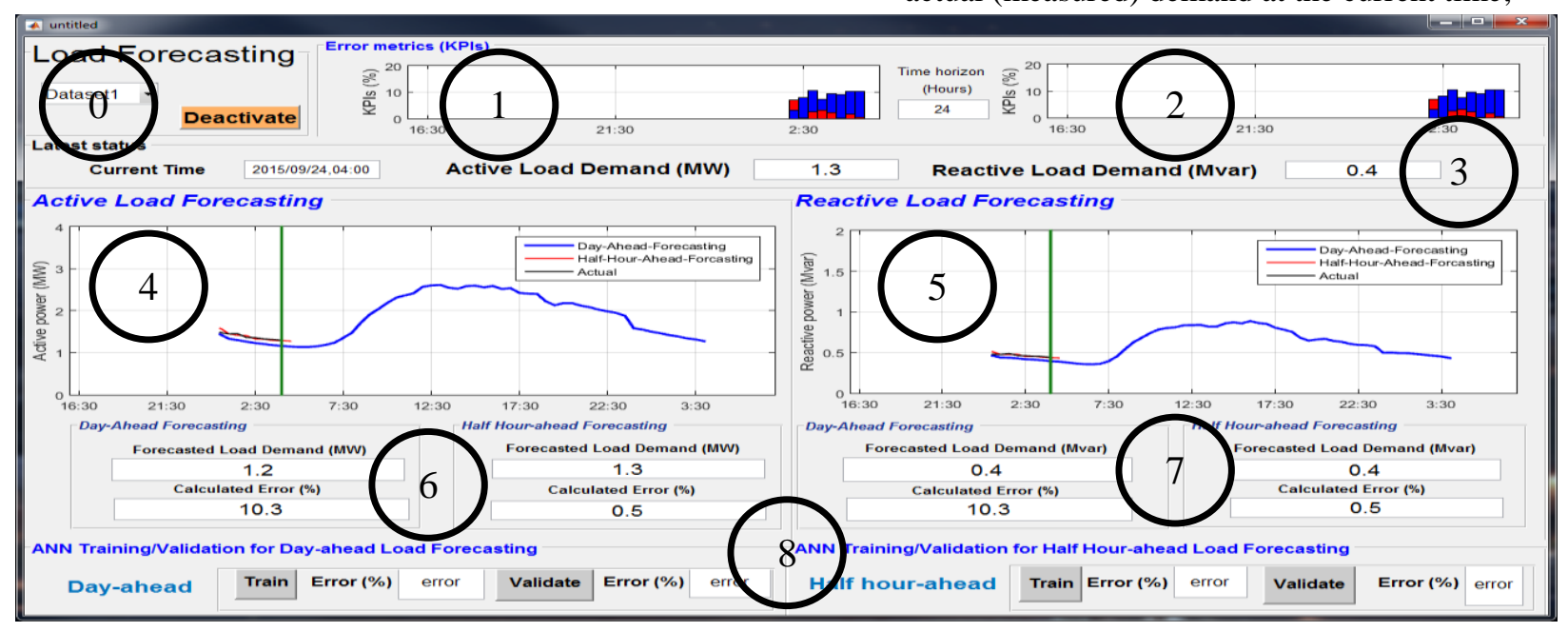

Figure 7. Presentation of the GUI for day ahead demand forecasting 


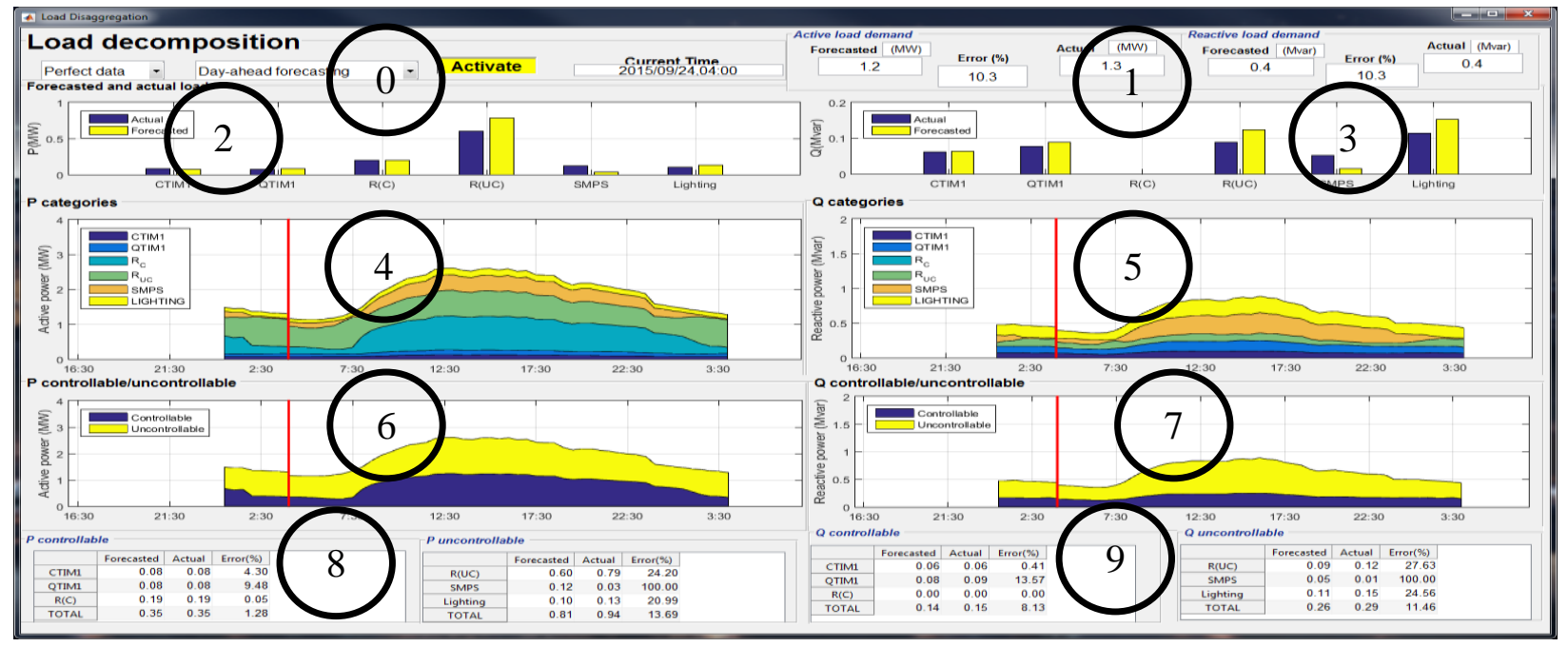

Figure 8. Presentation of the GUI for day ahead forecasting of demand composition

- 8: Training and validation of the ANN for day-ahead and half hour-ahead forecasting, respectively. There is an optional "Train" button for the user, who may want to retrain the DFANN with the most recent (3 months long) historical data, while the 'Validate' button performs validation of the ANN, i.e. testing the ANN with training data and comparing the outputs with the ANN target. The retraining can also be done automatically, after a predefined time period, e.g. every day.

Fig. 8 represents the GUI for DD, where numbered parts of the interface shown have the following function/meaning:

- 0: The drop-down menus offer different datasets (in this case "perfect data", i.e. data with no missing samples was chosen) and half hour or day-ahead forecasting. The Activate/Deactivate button serves to start or interrupt the GUI. Current time is also shown here.

- 1: Values of relative errors for active and reactive total demand forecasting (showing numerical values of the DF outputs for the current time);

- 2 and 3: Bar plots showing the forecasted (yellow) and actual (blue) demand of individual load categories, within total real and reactive demand, respectively.

- 4 and 5: Diagrams showing forecasted demand composition based on the forecasted total active and reactive demand (illustrated in Fig. 7) on the right side of the red vertical line, and actual demand composition, based on measured total active and reactive demand, on the left side of the line. The red line refers to the current time. Similarly to the case of DF, the curves move in real time to the left, together with the time labels, while the red line stays fixed. Also, the curves will move with the same time resolution as the resolution of the measurement data, with some time delay.
- 6 and 7: Diagrams showing forecasted controllable and uncontrollable load (on the right hand side of the vertical red line) based on the forecasted total active and reactive demand, and historical-actual controllable and uncontrollable load, based on measured total active and reactive demand. These curves move accordingly.

- 8 and 9: The values represent the forecasted and actual active and reactive demand, respectively, of individual load categories, and controllable/uncontrollable load. These are followed by the corresponding relative errors.

\section{CONCLUSION}

The main purpose of advanced demand profiling and the GUI is to support day ahead and half hour ahead DR planning, as it provides information about the flexibility potential of the demand-side over different times of the day. This information reduces the uncertainty of the actual (available) flexibility of the end-users, even before a DR signal is sent to them by their electricity supplier. The variety of information provided by the GUI can be further adjusted based on the specific requirements of the user. Furthermore, information about the types of flexible load (motors, heaters, lighting, etc.) that could be potentially shifted to different time (disconnected) enables a proper stability analysis before any kind of load shifting or load curtailment is performed. The introduced graphical representation of demand-side flexibility at the local (substation or other aggregating point) level can be further extended to a whole network and presented in "geographical map format" showing, in both time and space, the varying flexibility of the load at each individual substation (bus) in the network at any given point in time. The developed GUI for demand forecasting and demand decomposition, as a means of representing demand size and its flexibility in a user friendly 
way, can be used in a control room by the distribution system operator, who will be able to forecast, with high confidence, when, where (at which buses) and how much the demand can participate in, and increase the flexibility of, the low carbon network daily operation.

\section{ACKNOWLEDGMENT}

This research is supported by the EU Horizon 2020 project "Nobel Grid", contract number 646184.

\section{REFERENCES}

[1] D. S. Callaway and I. A. Hiskens, "Achieving controllability of electric loads," Proceedings of the IEEE, vol. 99, pp. 184-199, 2011.

[2] "Demand Side Flexibility Annual Report 2016 - Power Responsive," National Grid, 2016.

[3] K. Samarakoon, J. Ekanayake, and N. Jenkins, "Reporting Available Demand Response," Smart Grid, IEEE Transactions on, vol. 4, pp. 1842-1851, 2013.

[4] M. Pipattanasomporn, M. Kuzlu, S. Rahman, and Y. Teklu, "Load Profiles of Selected Major Household Appliances and Their Demand Response Opportunities," IEEE Transactions on Smart Grid, vol. 5, pp. 742-750, 2014.

[5] I. Richardson, M. Thomson, D. Infield, and C. Clifford, "Domestic electricity use: A high-resolution energy demand model," Energy and Buildings, vol. 42, pp. 1878-1887, 2010.

[6] K. Li, J. Ponoćko, L. Zhang, and J. V. Milanović, "Methodology for Close to Real Time Profiling of Aggregated Demand Using Data Streams from Smart Meters," presented at the 10th Mediterranean Conference on Power Generation, Transmission, Distribution and Energy Conversion (Med Power), Belgrade, Serbia, 2016.

[7] J. Ponoćko and J. V. Milanović, "Application of data analytics for advanced demand profiling of residential load using smart meter data," in PowerTech, 2017 IEEE Manchester, 2017, pp. 1-6.

[8] Y. Xu and J. V. Milanović, "Accuracy of ANN based methodology for load composition forecasting at bulk supply buses," in Probabilistic Methods Applied to Power Systems (PMAPS), 2014 International Conference on, 2014, pp. 1-6.

[9] Y. Xu and J. V. Milanović, "Day-Ahead Prediction and Shaping of Dynamic Response of Demand at Bulk Supply Points," IEEE Transactions on Power Systems, vol. 31, pp. 3100-3108, 2016.

[10] Y. Xiang, L. Junyong, Y. Wei, and C. Huang, "Active energy management strategies for active distribution system," Journal of Modern Power Systems and Clean Energy, vol. 3, pp. 533-543, 2015. 